\title{
A COMPARATIVE STUDY AMONG THREE REGISTRATION ALGORITHMS: PERFORMANCE, QUALITY ASSURANCE AND ACCURACY
}

\author{
D. Wujanz ${ }^{1}$, L. Barazzetti ${ }^{2}$, M. Previtali², M. Scaioni ${ }^{2}$ \\ ${ }^{1}$ technet GmbH, Am Lehnshof 8, 13467 Berlin, Germany - email: daniel.wujanz@ technet-gmbh.com \\ ${ }^{2}$ Dept. of Architecture, Built environment and Construction engineering (ABC) \\ Politecnico di Milano, Via Ponzio 31, Milan, Italy - emails: (luigi.barazzetti, mattia.previtali, marco.scaioni)@ polimi.it
}

\section{Commission II}

KEY WORDS: Accuracy, Automation, Least Squares, Registration, Terrestrial Laser Scanning.

\begin{abstract}
:
A critical task in every terrestrial laser scanning project is the transformation (addressed to as registration or alignment) of multiple point clouds into a common reference system. Even though this operation appears to be a solved and well-understood problem, the vast majority of available techniques still lack meaningful quality measures that allow the user to understand and analyze the final outputs. The erroneous estimation of registration parameters may cause systematic biases that falsify those subsequently outcomes such as deformation measurements on historical buildings, CAD-drawings of individual elements, or 3D models devoted to analyze the verticality of a tower. Thus, this article compares three common registration algorithms, namely target-based registration, the Iterative-Closest Point algorithm (ICP) as well as a plane-based approach on examples related to different case studies concerning historical buildings.
\end{abstract}

\section{INTRODUCTION}

Digital reconstruction of complex architectures requires fast and reliable measuring tools able to capture the visible surfaces with millimeter-level accuracy. Terrestrial laser scanning (TLS) technology has become an essential tool for the generation of traditional deliverables in the architecture, engineering and construction (AEC) industry, such as 2D drawings (plans, sections and elevations), 3D models based on NURBS or meshes, and Building Information Models (BIM). Even though discussions about the accuracy of laser scanning are usually conducted in millimeters, its error budget is not restricted to the rangefinder accuracy.

Figure 1 illustrates the typical stages of a TLS project, which are represented on the horizontal axis. The first vital stage is planning, including the selection of a suitable laser scanner instrument that may offer sufficient precision and may feature an operating range to cover the entire scene. Concurrently, the choice of a geo-referencing strategy directly influences the configuration of the instrument viewpoints (Soudarissanane et al., 2011), together with the accessibility of the project area (Giussani and Scaioni, 2004). The second stage is data acquisition followed by primary data processing, which includes filtering the point clouds as well as transforming multiple point clouds into a common coordinate system, which is also referred to as (co-) registration or alignment. Finally, the desired deliverables are generated during secondary data processing. In Figure 1 two runs are illustrated that denote the chance of influencing the quality of the final outcomes of the TLS surveying process (green line), as well the related cost of change (red line). It is evident that errors that occur in one of the initial stages require actions that for their mitigation whose cost rises up as far as the project carries on. Hence, it is desirable to identify errors in the early stage, possibly.

In such a context, the evaluation of the registration quality plays a fundamental role in the delivery of accurate technical products at the end of the TLS surveying project.

As standard architectural representation scales range from 1:20 to 1:100 (notwithstanding scale 1:50 is still the reference for several projects in the field of cultural heritage documentation), scans must be registered with very high metric quality. For instance, in the case of deliverables at a scale 1:20, registration accuracy cannot exceed the 4-6 $\mathrm{mm}$ range between adjacent scans, which is a value similar to the nominal precision of most phase shift laser scanners used for architectural surveying. However, it is well known that the registration error accumulates. Increasing the number of scans could result in error propagation exceeding the aforementioned required registration accuracy.

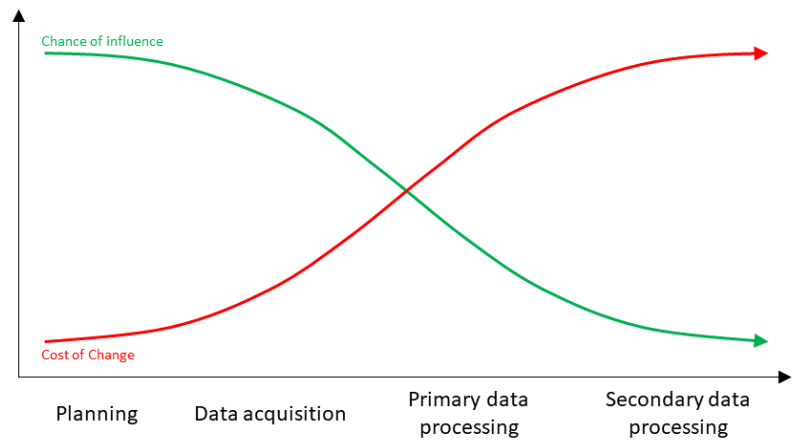

Figure 1. Typical stages of a terrestrial laser (TLS) scanning project.

Even though registration appears to be a solved and wellunderstood problem, the vast majority of available solutions still lack meaningful quality measures that allow the user to draw sound conclusions about the final outputs. Erroneous registration parameters may cause systematic biases that hence falsify subsequently generated results. Thus, this article compares three common registration algorithms, namely target-based registration, the Iterative-Closest Point algorithm (ICP) as well as a plane-based approach on examples related to different case studies concerning historical buildings. One of the most critical aspects in the registration process is the relation between the achieved accuracy and the corresponding quality measures. Hence, our study analyses the transparency of the quality 
measures for all considered algorithms. Furthermore, we compare the achieved accuracy with respect to a reference established by a geodetic network.

\section{REGISTRATION STRATEGIES}

In general, the strategies for transforming all scans collected within a TLS project into a common reference frame may be distinguished in two main categories: georeferencing and registration/alignment approaches (see Vosselman and Maas, 2010). The peculiarity of the first method is that the common coordinate frame is set up by a reference coordinate system which is established by geodetic measurements (by means of theodolites or GNSS instruments) or additional sensors embedded into upto-date laser scanners (e.g., GNSS and levelling sensors). The second methodology works solely on the acquired data and will be of interest in the following sections.

\subsection{Target-based registration}

A quite common way of transforming several scans into a reference coordinate system is the use of artificial targets (Chow et al., 2010). Therefore, spherical, cylindrical or planar targets are used, which have to be placed within the scene. The target locations must be chosen in a way that they are visible from at least two viewpoints that should be registered. After data acquisition, the target centers need to be determined either by computing the center point of the sphere through adjustment calculation or by using appropriate algorithms (Abmayr et al., 2008). Regarding the target configuration, it is important to mention that targets should be located in a way that they are distributed in all cardinal directions and not collinear. After extracting the target centers, point to point correspondences may be established between individual points from different viewpoints. Based on this information transformation parameters among the two viewpoints can be computed and finally applied to the corresponding point clouds obtaining a registered dataset (Scaioni, 2012).

Several disadvantages can be associated with this strategy such as (i) the effort of distributing the targets in the area of interest, (ii) the limited use of the inherent redundancy within the overlapping region, and (iii - optionally) the required survey of the targets in order to determine their 3D-coordinates in a reference system. A general assumption of this approach is that the majority of targets remain geometrically stable throughout the survey campaign. However, this might not be the case, especially in public spaces. The reason why targets are still commonly used in practice despite its numerous drawbacks can be found in the fact that all well-established operational procedures for total stations are directly transferable to TLS.

\subsection{Surface-based registration (ICP)}

The most versatile registration method uses redundantly captured regions of two point clouds and forms the family of surface-based registration algorithms. A substantial advantage of this strategy over target-based registration is the actual use of the redundant information in the overlapping regions of two or more point clouds. A drawback of these surface-based algorithms is their dependence to a sufficient pre-alignment of two datasets, which is required to trigger the iterative registration process.

Three general options can be used to satisfy this requirement namely (i) manual determination of a few correspondences, (ii) measurement of the individual location and orientation of two scans by additional geodetic sensors, which today may be frequently found in up-to-date laser scanners, and (iii) use of prealignment algorithms, such as Aiger et al. (2008).
After the relative coarse alignment of two data sets, fine matching may be conducted. The necessity of coarse alignment stage before fine matching can be explained by the non-convexity of the optimization function that leads to a convergence region in which the two point clouds need to fall into to avoid local minima of the target function during parameter estimation. One of the first surface-based registration algorithms, named the iteratively closest point algorithm (ICP), has been proposed by Besl and McKay (1992) where point-to-point correspondences are established. This step has to be rated critical because nonrepeatable points are captured by TLS, notwithstanding this issue can be compensated to a certain degree by establishing pointtriangle correspondences (Chen and Medioni, 1991). Several successive modified versions of ICP have been proposed, as reviewed by Rusinkiewicz and Levoy (2001).

In the pairwise registration of two scans, one (called 'reference' or 'master') is kept fixed, while the other ('target' or 'slave') is transformed to match the reference. In general, the ICP algorithm iterates over two steps until a convergence criterion is satisfied:

1. Find correspondence set $\boldsymbol{K}=\{(\boldsymbol{p}, \boldsymbol{q}) \boldsymbol{\}}$ from 'target' point cloud $\mathbf{P}$, and 'reference' point cloud $\mathbf{Q}$ transformed with current transformation matrix $\mathbf{T}$. The transformation matrix $\mathbf{T}$ is generally defined as a combination of a rigid body rotation and translation.

2. Update the transformation matrix $\mathbf{T}$ by minimizing an objective function $\mathbf{E}(\mathbf{T})$ defined over the correspondence set $\mathbf{K}$.

It is well known that ICP-based approaches are sensitive against deformed regions or changes of the object space between two scans, which yields in erroneous registrations (Wujanz, 2012). This fact may become a severe problem when laser scan registration has to be used to compare point clouds to detect deformations and changes (Scaioni et al., 2013; Lindenbergh and Pietrzyk, 2015).

\subsection{Plane-based registration}

Apart from using points for the sake of registration, detected objects can be used in the form of geometric primitives, such as planes (Previtali et al., 2014; Technet, 2018). As a first step, planar segments have to be extracted from the original point clouds (Vosselman et al., 2004) while for each segment plane parameters are adjusted. Then, correspondences among identical planes are computed instead of iteratively matching single points between scans, such as in surface-based approaches. By using adjusted planes instead of points, the precision of the resulting transformation parameters notably increases. Geometric features in the form of planes can be numerously found in nearly all manmade structures and are hence particularly suitable for registration of data captured on historical buildings especially for the documentation of indoor environments (Previtali et al., 2018).

\section{COMPARISON ON ARCHITECTURAL CASE STUDIES}

Two case studies are illustrated and discussed in this section. The laser scanner used for all applications was a Faro Focus 3D HDR X130 (www.faro.com), which features a distance precision of \pm 2 $\mathrm{mm}$ and is suitable for architectural surveying. Laser scans were acquired to produce plans, sections, elevations at the scale 1:50, whereas details required a high level of detail at the scale 1:20. 


\subsection{Case Study 1: the Chiaravalle Abbey}

Digital documentation required a set of scans of the cloister and refectory of the Chiaravalle Abbey (Fig. 2), located in proximity of Milan, Italy. A set of 24 scans was acquired in half a day, using a scans resolution variable between 28 and 44 million points/scan. Scans were acquired starting from the refectory, obtaining a sequence of five scans along a line that forms an uncontrolled network scheme. The first consideration is therefore related to the need to register scans which do not have an optimal geometric configuration and could result in error propagation in a way similar to an open traverse in topographic surveying (see Schofield and Breach, 2007). Network geometry could be simply improved by taking scans without moving the scanner along a straight line.
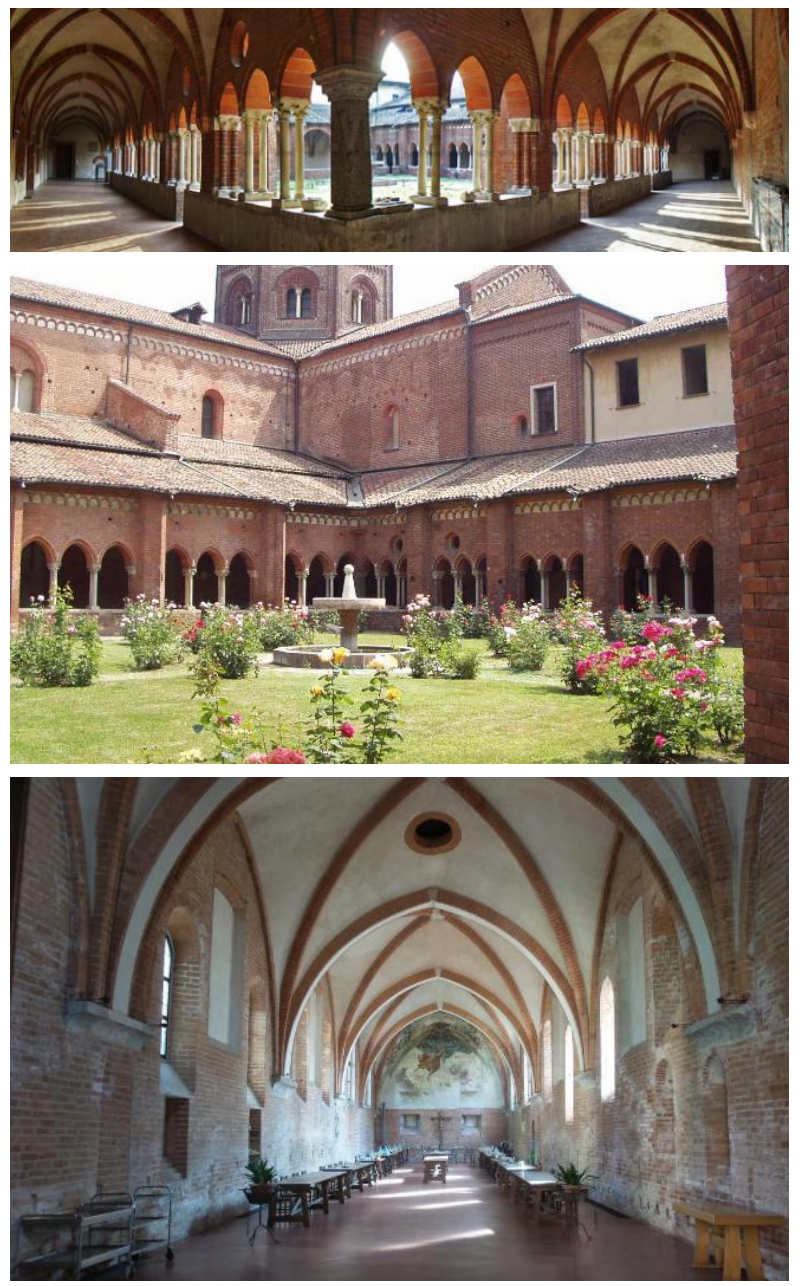

Figure 2. Some images of the cloister and refectory of the Chiaravalle Abbey (Milan, Italy).

On the other hand, data acquisition must take into consideration the aim of the survey, which was an accurate reconstruction of the historic vaults. Scans were acquired placing the scanner in the middle of each vault to capture points homogenously distributed on the intrados and ribs as well as on the walls. After acquiring the scans in the refectory, a set of 15 scans was taken around the four sides of the cloister. The geometry forms a closed loop connected to the previous five scans through a scan in front of the refectory door. Finally, four scans were taken in the central part of the cloister to capture the external facades.

\subsection{Case Study 2: Albergo Diurno di Porta Venezia}

The second case study is the Albergo Diurno di Porta Venezia in Milan (Fig. 3), which is an underground Art Nouveau space used starting from 1926 but now abandoned. It features several shops (barber, hairdresser, manicure, etc.) organized in a large entrance, connected to several bathrooms for travelers or inhabitants (Fig. 4). The particular geometry of the Albergo Diurno required a set of 43 scans: outside, in the main entrance and along two narrow parallel corridors. The instrument used was a Faro Focus 3D HDR X130, set up at geometric resolutions resulting in scans made up of 44, 28, and 11 million points each. Data acquisition was integrated with total station measurements to better control the network geometry.
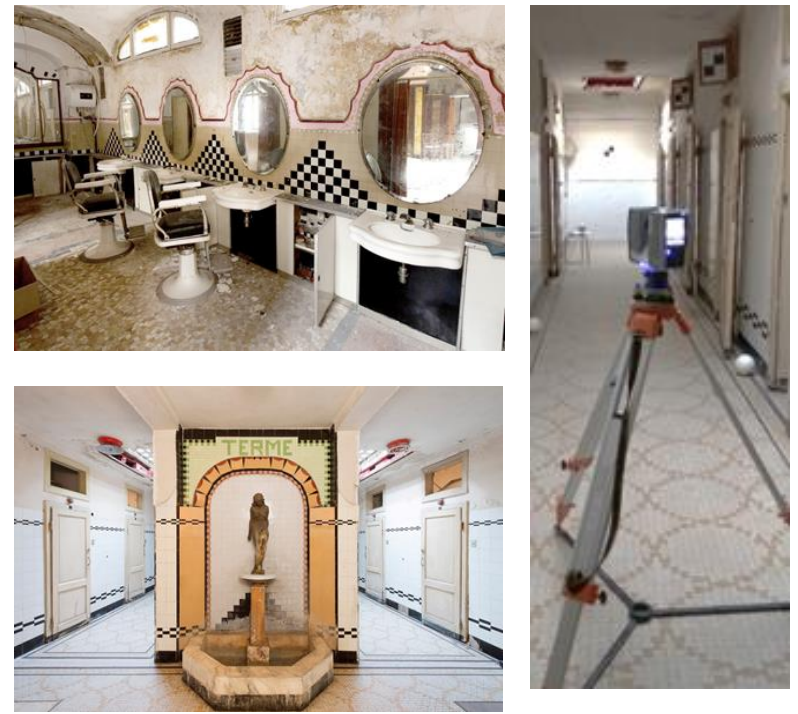

Figure 3. Some images of the Albergo Diurno di Porta Venezia (Milan, Italy).

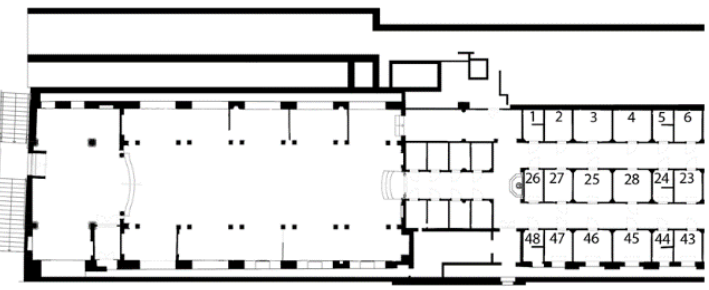

Figure 4. Planimetric view of the Albergo Diurno di Porta Venezia.

\section{RESULTS}

This section introduces the results generated using different solutions for scan registration illustrated in Section 2 along with those data sets (Case Studies 1 and 2) already discussed in the previous section. The following software packages were used:

- $\quad$ Faro Scene 7 (Faro, 2018); and

- Technet Scantra 2.3 (Technet, 2018).

The reference data in for Case Study 2 (Albergo Diurno di Porta Venezia) were represented by artificial targets measured by using a theodolite Leica Geosystems TCP1203 with a nominal accuracy of range measurements of $\pm 2 \mathrm{~mm}$. Leica Geosystems 
LGO 8 (Leica Geosystems, 2018) was then used to compute the network adjustment which also yields in individual stochastic measures. Case Study 1 (Chiaravalle Abbey) was processed using TLS data only.

Faro Scene 7 is a laser scan registration software that features an implementation of the ICP algorithm as well as the option to use artificial targets. Both strategies were individually applied in this study. As for any implementation of ICP, the outcome may be strongly influenced by the number of samples for which corresponding points are iteratively determined as well as a chosen search radius in which a corresponding potential points may be searched for. It is evident that the latter parameter has a notable influence on the final registration results. In addition, the search radius is closely coupled to the quality of the preliminary coarse alignment of the scans. One of the most important issues in processing any kind of sensed data is quality assurance. Wujanz (2012) reported in this context that the implemented quality measures in both academic and commercial implementations of the ICP are not meaningful. The most popular quality measure is the Root Mean Squared (RMS) of residuals, which interestingly can be arbitrarily set by the user. As a consequence, a user is not able to identify misalignments between point clouds based on analyzing the quality measures. Hence, it is advisable to visually inspect the result of ICP-registration.

Technet Scantra 2.3 is a laser scan registration software that is capable of introducing identical planes and points as well as inclinometer information as observations. The software works within three stages (Wujanz et al., 2018) namely:

1. plane detection;

2. pairwise registration (datum-independent); and

3. block adjustment (datum-dependent).

The first stage can be manipulated by specifying a certain required planarity threshold as well as a minimum number of points per plane. Apart from estimating the unknown parameters of the planes, stochastic measures are computed in correspondence of every entity. Scantra features various numerical and visual quality measures at different stages of the processing workflow. After pairwise registration the standard deviations of the estimated transformation parameters are reported. The quality depends on the applied scanner's noise, the individual planarity of detected planes, as well as their distribution within the object space. After the final Least-Squares block adjustment, datum-dependent quality measures are reported for every station considering the stochastic properties of all planes as well as the entire network scheme. This measure quantifies the accuracy of how well a station is connected to the reference station in form of a spatial standard deviation. Apart from that, misclosures between redundantly connected stations are reported as well as normalized residuals for every single registration.

\subsection{Case Study 1}

4.1.1 Plane-based registration. As discussed in Subsection 0, the chosen network scheme contains one area that is rather unfavorable. This part consists of five scans that form an uncontrolled open traverse while the stations are nearly collinear. To achieve a well-controlled network scheme, all stations were at least registered by two connections while two stations even featured six connections to adjacent scans. In total, 48 connections among all scans were established as depicted in Figure 5, where the refectory is on the left and the cloister on the right. Stations are represented by circles while lines depict registrations between the corresponding scans. The colours denote the estimated standard deviation of translations for two scans. Green lines means registrations with estimated shifts below $1 \mathrm{~mm}$ in terms of their standard deviation. Yellow lines highlight connections between 1 and $3 \mathrm{~mm}$. On average the standard deviation of the estimated translations sums up to $0.8 \mathrm{~mm}$ while the largest value is $2.4 \mathrm{~mm}$. Several registrations are highlighted by yellow lines indicating a comparably lower precision of the estimated transformation parameters. However, these connections are vital to control other registrations. Hence, it is not recommended to delete these connections.

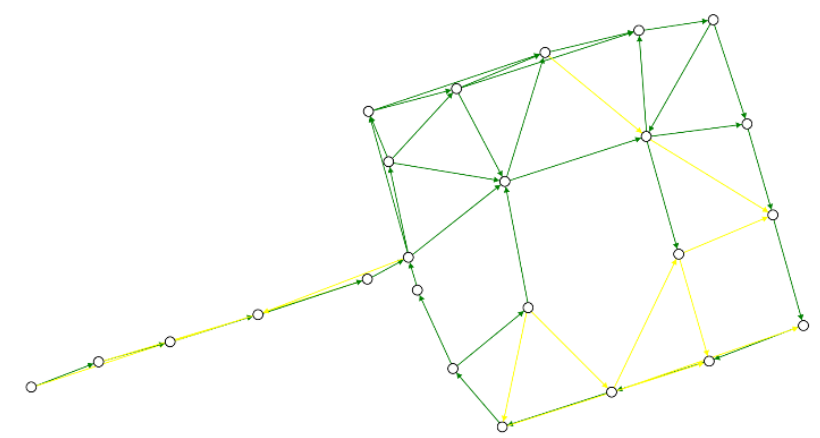

Figure 5. Case Study 1: results of pairwise plane-based registrations.

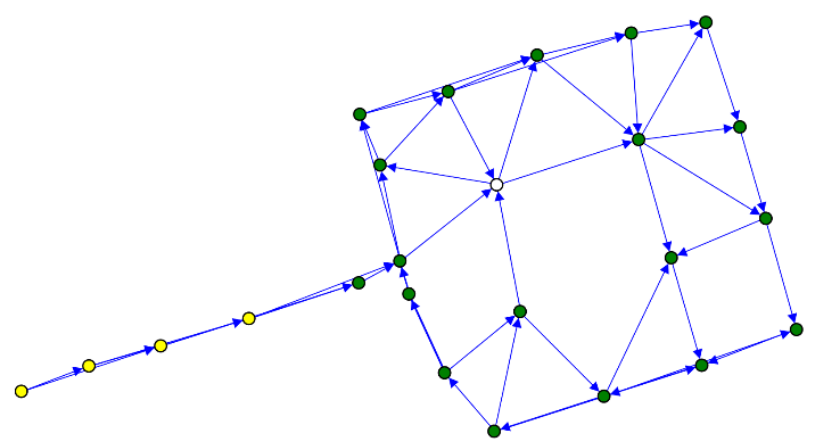

Figure 6. Case Study 1: datum-dependent quality measures after block adjustment.

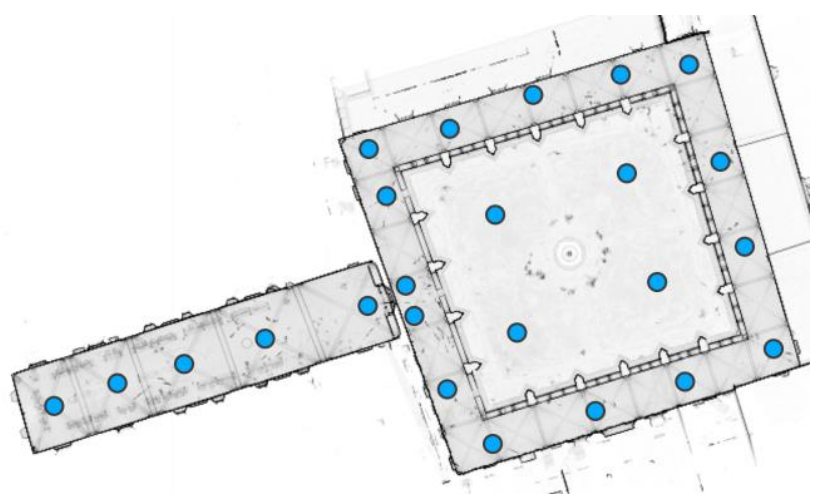

Figure 7. Case Study 1: Visualization of scan locations (blue circles) after registration. Similar results were obtained when using the three registration methods.

After pairwise registration, all stations are connected to one common data set. Subsequently, it is important to reveal potential conflicts between individual registrations which represents a vital task in quality assurance. This requires a redundant network configuration which is available in the present case. On average 
the misclosures after the block adjustment sum up to $1.1 \mathrm{~mm}$. In addition, datum-dependent quality measures are available, which were computed by means of variance-estimation. This step considers the stochastic properties of every detected plane as well as the entire network configuration. Figure 6 illustrates the outcome where green circles denote the spatial accuracy of a station up to $3 \mathrm{~mm}$, while yellow circles highlight stations with values larger than 3 and smaller than $10 \mathrm{~mm}$. The choice of the reference scan for registration plays an essential role in error propagation. Choosing the first scan on the outer left (Scan 0) as a reference station would result in error propagation reaching 0.0 $\mathrm{mm}$ for the reference and $16.0 \mathrm{~mm}$ for scan 14, which is located on the lower right. Setting Scan 21 as a reference, which is highlighted by a white inner circle in the upper center of the block, reduces error propagation to $8.0 \mathrm{~mm}$ for Scan 0, that becomes the scan registered with the lowest precision. The value is instead $2.7 \mathrm{~mm}$ for scan 14 . On average the absolute spatial accuracy sums up to $2.1 \mathrm{~mm}$. A final plan of the registered scans is shown in Figure 7.

4.1.2 Target-based registration. Registration of the scans by using a target-based approach was applied by using the software Faro Scene 7. Scan 21 was set as reference like in the previous plane-based solution (Par. 4.1.1). Figure 8 reports the error matrix for the registration of Case Study 1 data set. In particular, the matrix reports the pairwise registration error between scans. Each element $\mathrm{E}_{\mathrm{ij}}$ reports the registration error between scan $i$ and scan $j$. In this case it can be observed that most of the elements in the matrix are close to the main diagonal, meaning there is a limited target overlap between the scans. Elements far away from the main diagonal, like the elements $\mathrm{E}_{6,19}$, are representing the closure of the loop around the cloister. It can be observed that in this case, results shows a good registration between scans. The obtained final scan locations are very close to the one shown for the target-based registration in Figure 7.

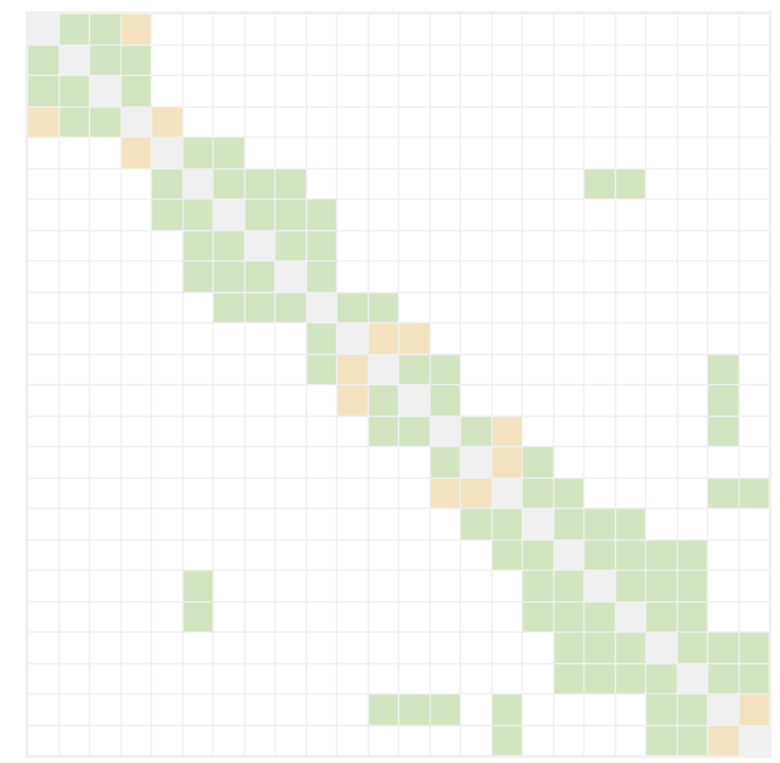

Figure 8. Case Study 1: matrix showing the results of scan pairwise registration using a target-based approach. Average target residuals are displayed using following colours: green $<3.0 \mathrm{~mm}$, orange between $3.0-10 \mathrm{~mm}$, red $>10$.

4.1.3 Surface-based registration. The surface-based method was carried out with the cloud-to-cloud option (ICP) in Faro Scene 7. Scan 21 was set as reference. The initial subsampling of the scans was set using a factor of $50 \mathrm{~mm}$. Registration statistics showed a mean precision of $2.9 \mathrm{~mm}$, whereas $64 \%$ of the matched scan pairs had an error less than $4 \mathrm{~mm}$. Minimum overlap was $22.9 \%$. An image of the error matrix for the Chiaravalle registration is shown in Figure 9. In this case, the scan locations are still very close to the one displayed in Figure 7. A $3 \mathrm{D}$ view of the registered scan is shown in Figure 10.

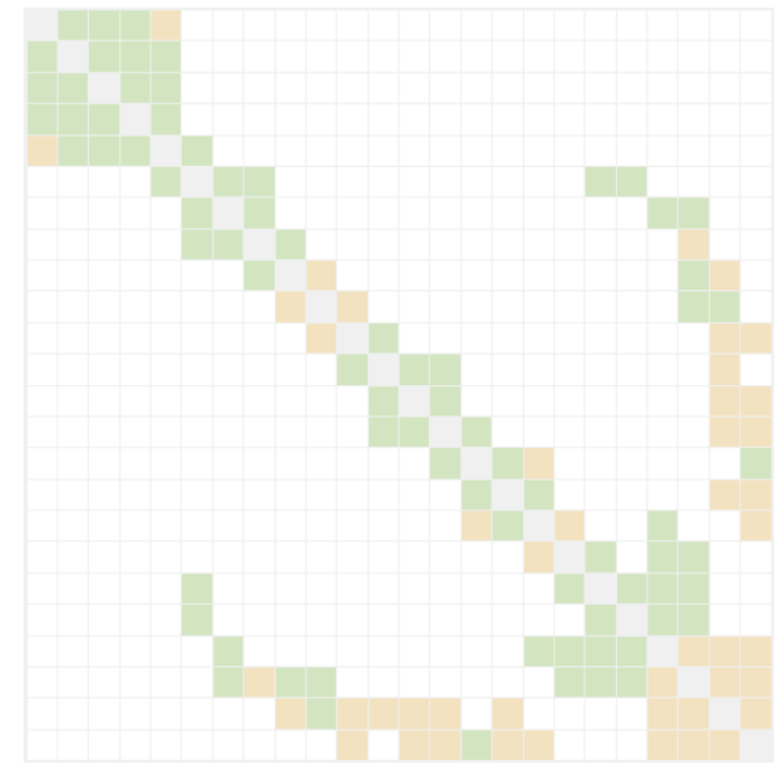

Figure 9. Case Study 1: matrix showing the results of scan pairwise registration using a surface-based approach. Average target residuals are displayed using the same colours adopted in Figure 8.

4.1.4 Comparison of registration methods. The use of the same scan (\#21) to establish the reference system allows a direct comparison of the computed scan locations. The reference system has its center in the origin of Scan \#21 and its horizontal direction angle equals zero. The $\mathrm{Z}$ axis is vertical. Graphs in Figures 11 and 12 show the difference for $\mathrm{X}, \mathrm{Y}$, and $\mathrm{Z}$ coordinates as obtained from different registration methods. The plane-based method was used as reference, and the variation was computed as $\mathrm{DX}=\mathrm{X}_{\text {plane }}-\mathrm{X}_{\text {target/surface. }}$. The same procedure was applied to $\mathrm{Y}$ and $\mathrm{Z}$ coordinates.

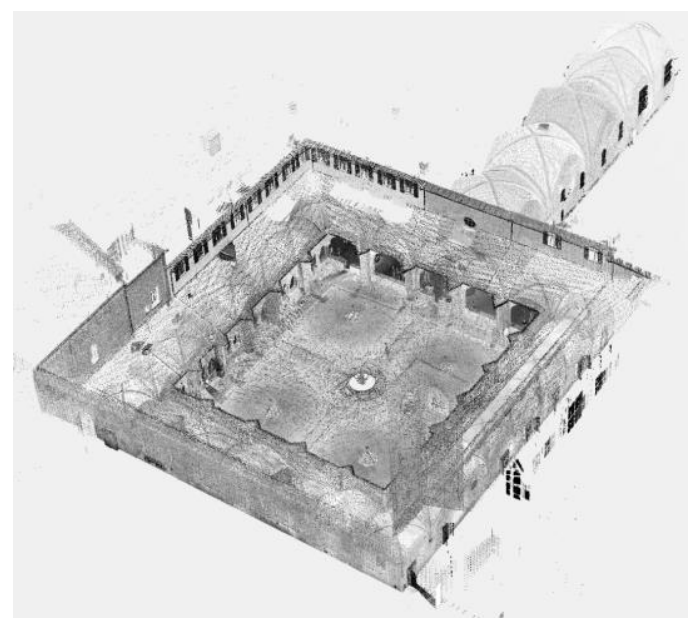

Figure 10. Case Study 1: 3D view of the registered scan with the surface-based method. 
The comparison between the plane- and target-based methods shows that the error increases moving towards the first scans. This error becomes significant especially for $\mathrm{X}$ and $\mathrm{Y}$, and it could be explained considering the network geometry. Indeed, these scans were those acquired in the refectory, where the geometry becomes that of an uncontrolled open traverse. Another consideration is related to the largest differences along $\mathrm{Z}$ for those scans between \#11 and \#15. This effect is larger for the comparison based on targets, notwithstanding the comparison with the surface-based method reveals a similar result.

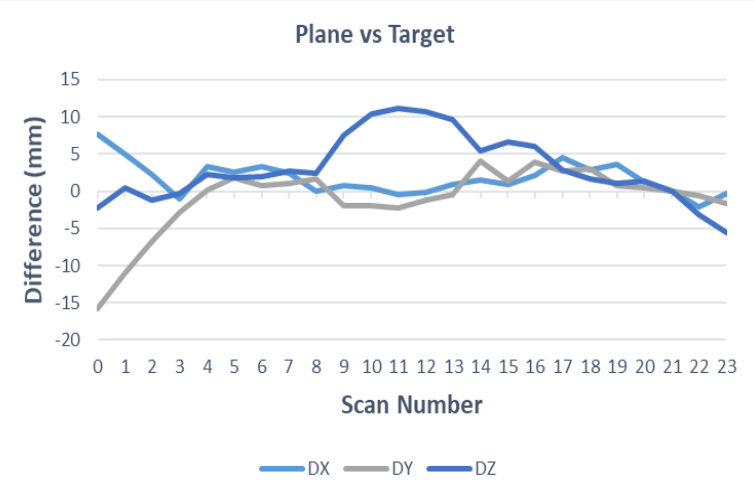

Figure 11. Case Study 1: differences on the computed scan locations between the plane- and target-based methods.

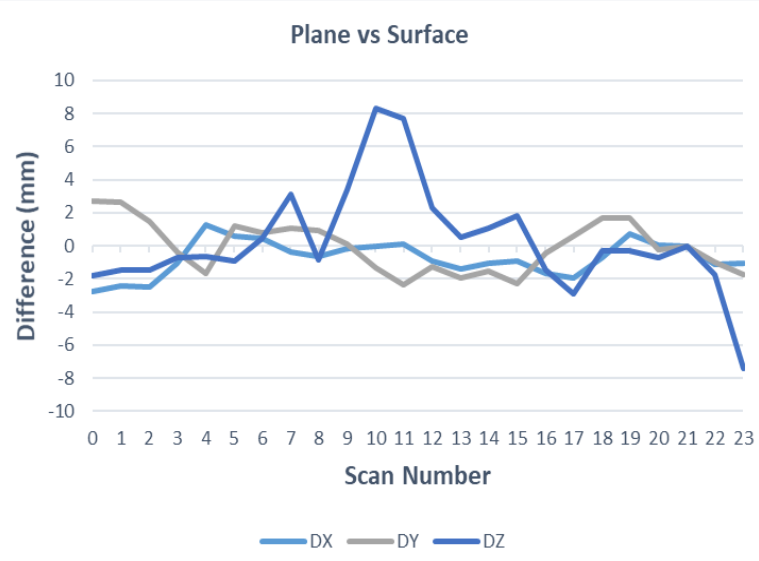

Figure 12. Case Study 1: differences on the computed scan locations between the plane- and surface-based methods.

\begin{tabular}{|c|c|c|c|}
\hline \multicolumn{5}{|c|}{ Plane-based vs target-based registration } \\
\hline & DX $(\mathrm{mm})$ & DY $(\mathrm{mm})$ & DZ $(\mathrm{mm})$ \\
\hline Mean & 1.7 & -1.0 & 3.1 \\
\hline Std.dev. & 2.2 & 4.5 & 4.5 \\
\hline Min & -2.1 & -15.9 & -5.6 \\
\hline Max & 7.7 & 4.0 & 11.2 \\
\hline Plane-based vs surface-based registration \\
\hline Mean & -0.7 & -0.1 & 0.2 \\
\hline Std.dev. & 1.0 & 1.5 & 3.2 \\
\hline Min & -2.8 & -2.4 & -7.4 \\
\hline Max & 1.3 & 2.7 & 8.3 \\
\hline
\end{tabular}

Table 1. Case Study 1: statistics on the differences of scan locations computed with three registration methods.
Table 1 shows the statistics (mean, standard deviation, max, and min) computed for DX, DY, and DZ. It is interesting that the surface- and plane-based result seem to be more consistent, with a minimum value for the mean that indicates the absence of systematic effects. The standard deviation of the differences between the surface- and plane-based methods is about 2-3 times better than target- and plane-based methods. Probably, the results obtained with the ICP and plane-based algorithm are more accurate than those achieved by using targets in the scene.

\subsection{Case Study 2}

The elongated geometry of Case Study 2 (Albergo Diurno di Porta Venezia) made necessary the use of theodolite measurements to control scan registration. The coordinates of some chessboard targets were therefore measured and incorporated in the adjustment. The scheme of the topographic networks with estimated errors ellipses is shown in Figure 13. In such a case, the ICP (surface-based) registration was omitted because of the poor overlap between adjacent scans.

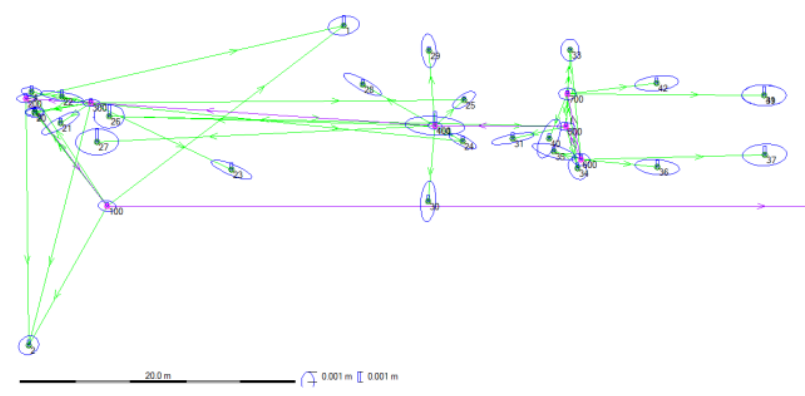

Figure 13. Case Study 2: scheme of the geodetic network with error ellipses of point coordinates.

4.2.1 Plane-based registration. The 43 stations of this data set were related by 87 connections to avoid uncontrolled registrations. On average the standard deviation of shifts for pairwise registrations sums up to $0.4 \mathrm{~mm}$, while the maximum value is $2.1 \mathrm{~mm}$. The last-mentioned value can be found on the outer left of Figure 14, which has been highlighted by the yellow line. The reason for the increased standard deviation may be found in a relatively small and unfavorable overlap between the two scans that connect the upper and lower level of the scene with a set of stairs. After the block adjustment misclosures between controlled registrations sum up to $0.5 \mathrm{~mm}$, which confirms the achieved high level of inner accuracy of the pairwise registration stage. To evenly distribute datum-dependent effects, a station roughly in the center of the network was chosen as a reference station, highlighted by a white inner circle in Figure 15. Regarding the datum-dependent measures, the absolute spatial accuracy is $1.3 \mathrm{~mm}$ on average while the largest value is $3.1 \mathrm{~mm}$ highlighted by one of the yellow inner circles.

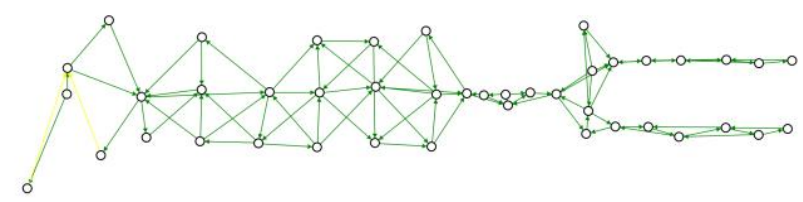

Figure 14. Case Study 2: results of pairwise plane-based registrations. 


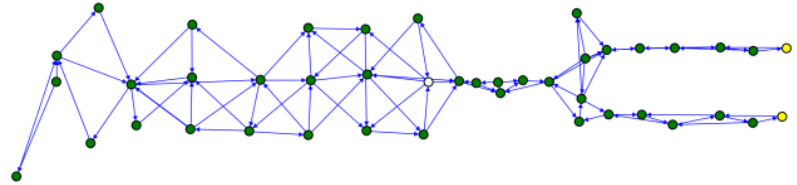

Figure 15. Case Study 2: datum-dependent quality measures after block adjustment.

4.2.2 Target-based registration. The 43 stations of this data set were registered with an average error of $4.0 \mathrm{~mm}$. The maximum horizontal and vertical errors were 13.8 and $9.1 \mathrm{~mm}$, respectively. The error matrix in Figure 16 shows that some scans (those at the beginning and end of the atrium and corridors) are connected to a large number of scans. The rightmost column corresponds to ground control points (GCPs) measured using a theodolite. An overall map of the computed scan location with Faro Scene is shown in Figure 17. Figure 18 Shows a 3D view of the registered scans of Case Study 2 data set.

4.2.3 Comparison of registration methods. Theodolite measurements give the reference system of this project. The comparison was carried out with the computation of the differences between coordinates obtained with both adopted methods: $\mathrm{DX}=\mathrm{X}_{\text {plane }}-\mathrm{X}_{\text {target }}$. The same procedure was performed for $\mathrm{Y}$ and $\mathrm{Z}$. The graph of the differences is shown in Figure 19, whereas statistics are reported in Table 2. Results confirm an accuracy of about 2-3 $\mathrm{mm}$ for $\mathrm{X}$ and $\mathrm{Y}$, whereas a larger error is found for $\mathrm{Z}$. The graph in figure 19 also shows that results are worse at the beginning of the sequence and for the last scans (from \#32 to \#42), which were acquired along the two corridors. Here, the effect of error propagation is significantly larger.

A final consideration on the problem in comparing the results with a superior coordinate system has to be mentioned. Targets give the reference system, and the accuracy of digitizing the targets in scans is usually too low (which is in the range of some millimeters). Also, the error budget of total station is also at the millimeter level. Such outcomes demonstrate that evaluating registration quality based on target is not the best solution to clarify which is the algorithm providing the best registration.

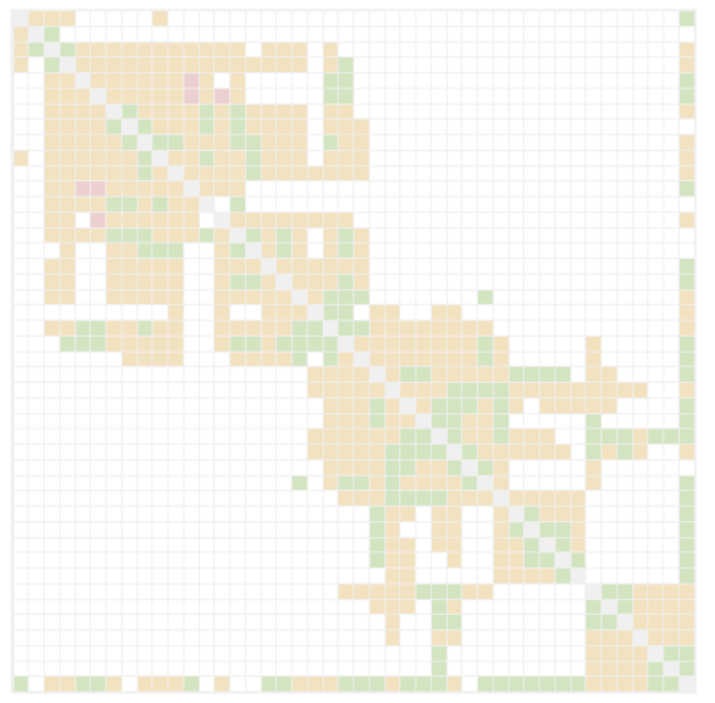

Figure 16. Case Study 2: matrix showing the results of scan pairwise registration using a target-based approach. Average target residuals are displayed using following colours: green $<3.0 \mathrm{~mm}$, orange between $3.0-10 \mathrm{~mm}$, red $>10$.

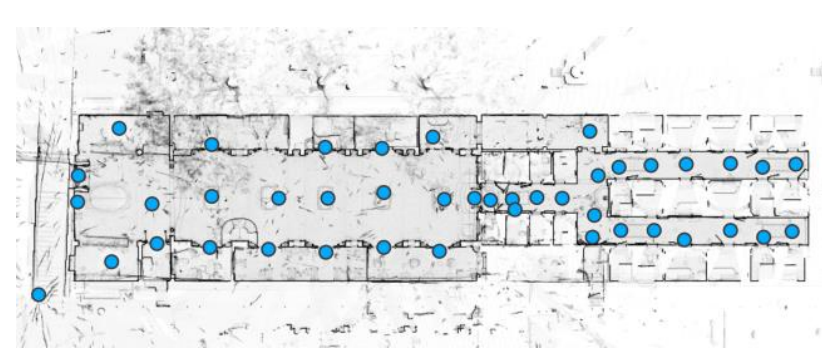

Figure 17. Case Study 2: Scan location computed with the targetbased method using Faro Scene software. Scans are overlaid over am existing plan of the Albergo Diurno di Porta Venezia.

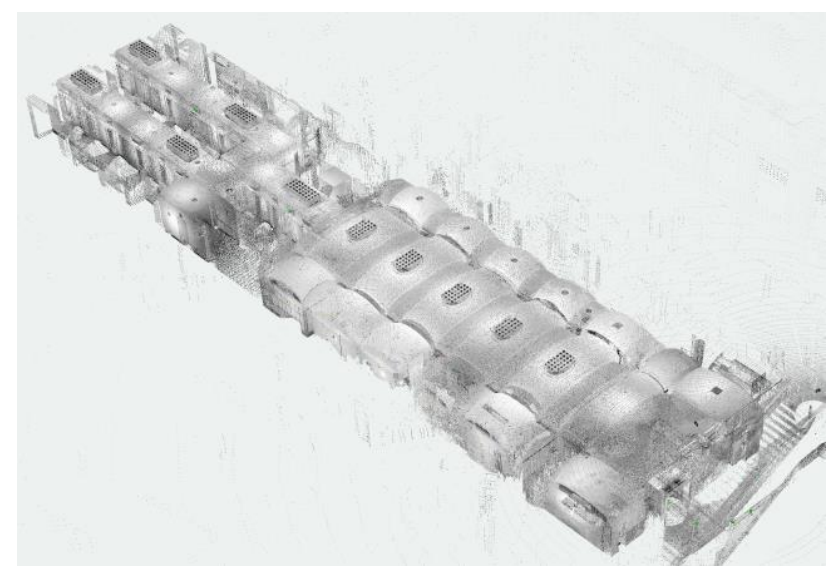

Figure 18. Case Study 2: 3D view of the registered scan with the target-based method.

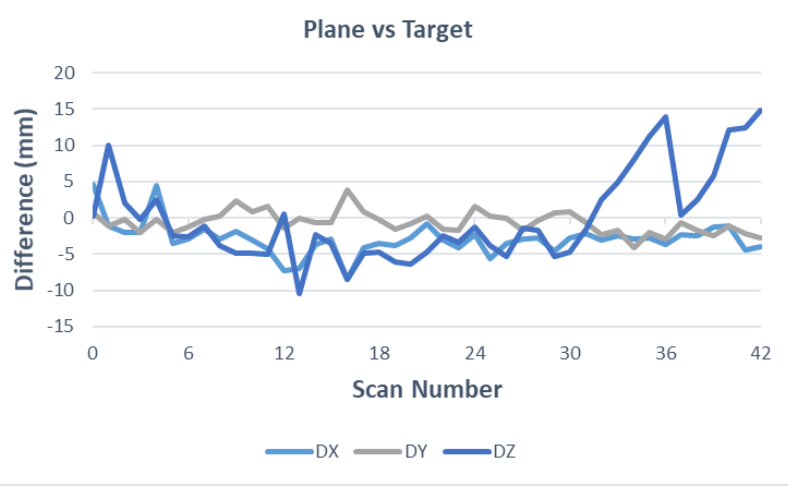

Figure 19. Case Study 2: differences on the computed scan locations between the plane- and target-based methods.

\begin{tabular}{|c|c|c|c|}
\hline \multicolumn{4}{|c|}{ Plane-based vs target-based registration } \\
\hline & DX (mm) & DY (mm) & DZ (mm) \\
\hline Mean & -2.9 & -0.7 & -0.1 \\
\hline Std.dev. & 2.3 & 1.5 & 6.3 \\
\hline Min & -8.5 & -4.1 & -10.5 \\
\hline Max & 4.6 & 3.8 & 14.9 \\
\hline
\end{tabular}

Table 2. Case Study 2: statistics on the differences of scan locations computed with two registration methods. 


\section{CONCLUSIONS}

Scan registration is a crucial task in digital documentation projects. Network geometry is often neglected during the acquisition of laser scans, forgetting that a good network scheme is necessary for a robust and reliable estimation of registration parameters. This paper has demonstrated that the use of registration algorithms able to provide a complete statistical output is a powerful solution to understand where largest registration errors are expected.

The use of both visual and statistical quality measures (such as those available in Technet Scantra) is a valid tool to support the evaluation of metric quality. Such visual tools are also useful to understand where the network geometry becomes weaker, considering that a network partially weak could have an impact on the subsequent production of final deliverables. As the acquisition phase is usually carried out considering the final output (e.g., scans taken in strategic locations to have a uniform distribution of points for production of measured drawings), knowing where such deliverables could be less accurate because of network issues is an additional advantage.

The comparison of three registration methods (plane-, targetand surface-based) has shown that redundant information derived from the scans may lead to superior accuracy with respect to target-based approaches, as demonstrated by the Case Study 1 (Chiaravalle Abbey, Milan). From a practical point-of-view, onsite work is even simplified because no preliminary distribution of targets in the scene is necessary. The use of plane-to-plane correspondences also reduces the effect of sampling uncertainty in surface-based methods (ICP).

Targets remain the best solution to register scans in a superior reference system. The case Study 2 (Albergo Diurno di Porta Venezia, Milan) has demonstrated that a few targets measured with a theodolite may notably stabilize the network geometry, providing additional information to check registration quality. However, a combined solution based on a limited targets in strategic location and planes may simplify on-site work and improve the overall metric accuracy.

\section{REFERENCES}

Abmayr, T., Härtl, F., Hirzinger, G., Burschka, D., Fröhlich, C., 2008. A correlation-based target finder for terrestrial laser scanning. J. App. Geod., Vol. 2(3):131-137.

Aiger, D., Mitra, N.J., Cohen-Or, D., 2008. 4-points congruent sets for robust pairwise surface registration. ACM TOG, Vol. 27(3), paper No. 85 .

Besl, P.J., McKay, N.D., 1992. A method for registration of 3-D shapes. IEEE Trans. PAMI, Vol. 14(2):239-256.

Chen, Y., Medioni, G., 1992. Object modelling by registration of multiple range images. Image Vision Comput., Vol. 10(3):145155.

Chow, J., Ebeling, A., Teskey, B., 2010. Low cost artificial planar target measurement techniques for terrestrial laser scanning. In: Proc. FIG Congress 2010, Sydney, Australia, 11-16 April 2010, 13 pages.

Faro, 2018. Faro Scene. Available from: https://www.faro.com/ products/product-design/faro-scene (last accessed on 20/12/2018).

Giussani A., Scaioni M., 2004. Application of TLS to Support
Landslides Study: Survey Planning, Operational Issues and Data Processing. Int. Arch. Photogramm. Remote Sens. Spatial Inf. Sci., Vol. 36, Part 8/W2, pp. 318-323.

Leica Geosystems, 2018. Leica Geo Office - one integrated Office Software. https://leica-geosystems.com/products/totalstations/software/ leica-geo-office (last accessed on 20/12/2018).

Lindenbergh, R., Pietrzyk, P., 2015. Change Detection and Deformation Analysis using Static and Mobile Laser Scanning. App. Geomat., Vol. 7: 65-74.

Previtali, M., Barazzetti, L., Brumana, R., Scaioni, M., 2014. Scan registration using planar features. Int. Arch. Photogramm. Remote Sens. Spat. Inf. Sci., Vol. 40, Part 5, 501-508.

Previtali, M., Díaz-Vilariño, L., Scaioni, M., 2018. Indoor Building Reconstruction from Occluded Point Clouds Using Graph-Cut and Ray-Tracing. Appl. Sci., Vol. 8(9), paper No. 1529.

Rusinkiewicz, S., Levoy, M., 2001. Efficient variants of the ICP algorithm. In: Proc. $3^{\text {rd }}$ Int. Conf. on 3-D Digital Imaging and Modeling, Quebec City, Quebec, Canada, 28 May-1 June 2001, pp. 145-152.

Scaioni, M., 2012. On the estimation of rigid-body transformation for TLS registration. Int. Arch. Photogramm. Remote Sens. Spatial Inf. Sci., Vol. 39, Part B5, pp. 601-606.

Scaioni, M., Roncella, R., Alba, M.I., 2013. Change Detection and Deformation Analysis in Point Clouds: Application to Rock Face Monitoring. Photogramm. Eng. Remote Sens., Vol. 79(5):441-456.

Schofield, W., Breach, M. 2007. Engineering Surveying - $6^{\text {th }}$ Edition. Butterworth-Heinemann.

Soudarissanane, S., Lindenbergh, R., Menenti, M., Teunissen, P., 2011. Scanning geometry: Influencing factor on the quality of terrestrial laser scanning points. ISPRS J. Photogramm. Remote Sens., Vol. 66(4):389-399.

Technet, 2018. Scantra - Beyond clouds. Available from: https://www.technet-gmbh.com/en/products/scantra (last accessed on 20/12/2018).

Vosselman, G., Gorte, B. G., Sithole, G., Rabbani, T., 2004. Recognising structure in laser scanner point clouds. Int. Arch. Photogramm. Remote Sens. Spat. Inf. Sci., Vol. 46, Part 8, pp. 3338.

Vosselman, G., Maas, H.-G., 2010. Airborne and Terrestrial Laser Scanning. CRC Press, 320 pages.

Wujanz, D., 2012. Towards transparent quality measures in surface based registration processes: Effects of deformation onto commercial and scientific implementations. Int. Arch. Photogramm. Remote Sens. Spat. Inf. Sci., Vol. 39, Part B5, pp. 251-256.

Wujanz, D., Schaller, S., Gielsdorf, F., Gründig, L., 2018. Planebased registration of several thousand laser scans on standard hardware. Int. Arch. Photogramm. Remote Sens. Spat. Inf. Sci., Vol. 42, Part 2, pp. 1207-1212. 